\title{
ON HOMOLOGY CLASSES REPRESENTED BY REAL ALGEBRAIC VARIETIES
}

\author{
JACEK BOCHNAK \\ Department of Mathematics, Vrije Universiteit \\ De Boelelaan 1081, $1081 \mathrm{HV}$ - Amsterdam, The Netherlands \\ E-mail: bochnak@cs.vu.nl \\ WOJCIECH KUCHARZ \\ University of New Mexico, Department of Mathematics and Statistics \\ Albuquerque, New Mexico 87131-1141, U.S.A. \\ E-mail: kucharz@math.unm.edu
}

Introduction. Let $X$ be a compact real algebraic variety (essentially, a compact real algebraic set, see Section 1$)$. Denote by $H_{k}^{\text {alg }}(X, \mathbb{Z} / 2)$ the subgroup of $H_{k}(X, \mathbb{Z} / 2)$ generated by the homology classes represented by Zariski closed $k$-dimensional subvarieties of $X$. If $X$ is nonsingular and $d=\operatorname{dim} X$, let $H_{\mathrm{alg}}^{k}(X, \mathbb{Z} / 2)$ be the subgroup of $H^{k}(X, \mathbb{Z} / 2)$ consisting of all the cohomology classes that are sent via the Poincaré duality isomorphism $H^{k}(X, \mathbb{Z} / 2) \rightarrow H_{d-k}(X, \mathbb{Z} / 2)$ into $H_{d-k}^{\text {alg }}(X, \mathbb{Z} / 2)$.

In this short paper we survey certain results concerning the groups $H_{k}^{\text {alg }}$ and $H_{\text {alg }}^{k}$, and their applications. Section 1 contains the precise definitions of these groups (based on a construction of the fundamental homology class of a compact real algebraic variety) and theorems establishing their functorial properties and relating them to the StiefelWhitney classes of algebraic vector bundles. With the exception of Theorem 1.7 (ii), all the results (modulo minor modifications) come from the classical source [15]. In Section 2 we adopt a scheme-theoretic point of view and discuss how the groups $H_{k}^{\text {alg }}$ are related to the theory of algebraic cycles, especially rational and algebraic equivalence. In particular, we give a purely algebraic geometric description of $H_{k}^{\text {alg }}$. Our main references are $[15,17$, $25,28]$. In Section 3 we study how the groups $H_{\mathrm{alg}}^{k}(X, \mathbb{Z} / 2)$, for $k=1,2$, vary as $X$ runs through the class of nonsingular real algebraic varieties diffeomorphic to a given closed $\mathcal{C}^{\infty}$ manifold. We rely mostly on $[9,11,37]$, but the reader may also wish to consult

Both authors were partially supported by NATO Collaborative Research Grants Programme, CRG 960011. The second author was partially supported by NSF Grant DMS-9503138.

1991 Mathematics Subject Classification: Primary 14C10, 14F25, 14P25.

Received by the editors: January 2, 1997; in the revised form: February 6, 1997.

The paper is in final form and no version of it will be published elsewhere. 
$[1,2,3,4,6,13,14,26,31,33,35,36]$ to see other results and the history of the subject and motivation for its development. It is, in general, very hard to compute $H_{\text {alg }}^{k}(X, \mathbb{Z} / 2)$ for a given $X$. In Section 4 we explain what is known for $k=1$ and $X=V_{\mathbb{R}}$, where $V$ is a nonsingular complex projective variety and $V_{\mathbb{R}}$ is its underlying real algebraic structure. The results are very satisfactory when $V$ is a complex elliptic curve. Our exposition follows $[7,12,19,22,23]$. We do not present computation of $H_{\mathrm{alg}}^{1}$ for other classes of real algebraic varieties; for this some good reference are $[29,30,31,35,36]$. In Section 5 we describe various applications of the groups $H_{k}^{\text {alg }}$ and $H_{\mathrm{alg}}^{k}$ contained in $[2,6,8,9,10,14]$. For other applications the reader may refer to $[2,4,6,13,14,26,32]$.

We do not strive to present the theory in its maximal generality. For example, we use the singular homology instead of the Borel-Moore homology and therefore have to assume compactness in the construction of the fundamental homology class of a real algebraic variety. Furthermore, few proofs are given since a complete exposition "from scratch" would require too much space.

1. Main general results. Throughout this paper the term real algebraic variety designates a locally ringed space isomorphic to a Zariski locally closed subset of $\mathbb{P}^{n}(\mathbb{R})$, for some $n$, endowed with the Zariski topology and the sheaf of real-valued regular functions. It is well known that every real algebraic variety is isomorphic (as a locally ringed space) to a Zariski closed subvariety of $\mathbb{R}^{n}$ for some $n$ [6, Proposition 3.2.10, Theorem 3.4.4] (note that real algebraic varieties defined above are called affine real algebraic varieties in [6]). Morphisms between real algebraic varieties will be called regular maps. Every real algebraic variety carries also the Euclidean topology, that is, the topology induced by the usual metric topology on $\mathbb{R}$. Unless explicitly stated otherwise, all topological notions related to real algebraic varieties will refer to the Euclidean topology.

Given a $d$-dimensional real algebraic variety $X$ with structure sheaf $\mathcal{R}_{X}$, a point $x$ of $X$ is said to be nonsingular if the local ring $\mathcal{R}_{X, x}$ is regular of Krull dimension $d$; if every point of $X$ is nonsingular, then $X$ is called nonsingular. The set $X^{0}$ of nonsingular points of $X$ is Zariski open in $X$ and $\operatorname{dim}\left(X \backslash X^{0}\right)<d$. Furthermore, $X^{0}$ is a $d$-dimensional $\mathcal{C}^{\infty}$ manifold and hence it follows from the excision property that

$$
H_{d}(X, X \backslash\{x\} ; \mathbb{Z} / 2) \cong \mathbb{Z} / 2 \text { for every } x \in X^{0} .
$$

THEOREM 1.1. Let $X$ be a compact d-dimensional real algebraic variety. Then there exists a unique homology class $[X]$ in $H_{d}(X, \mathbb{Z} / 2)$ such that for every nonsingular point $x$ of $X$ the image of $[X]$ in $H_{d}(X, X \backslash\{x\} ; \mathbb{Z} / 2)$, under the canonical homomorphism $H_{d}(X, \mathbb{Z} / 2) \longrightarrow H_{d}(X, X \backslash\{x\} ; \mathbb{Z} / 2)$, is a generator of $H_{d}(X, X \backslash\{x\} ; \mathbb{Z} / 2)$.

Proof. The quickest proof is via resolution of singularities. By [5, 20], there exist a compact nonsingular $d$-dimensional real algebraic variety $\tilde{X}$ and a regular map $\pi$ : $\tilde{X} \longrightarrow X$ such that $\pi: \tilde{X} \backslash \pi^{-1}\left(X \backslash X^{0}\right) \longrightarrow X^{0}$ is a biregular isomorphism. Denote by $[\tilde{X}]$ the fundamental $\mathbb{Z} / 2$ homology class of the closed $\mathcal{C}^{\infty}$ manifold $\tilde{X}$. Obviously, the homology class $[X]=H_{d}(\pi)([\tilde{X}])$ has the required property. The uniqueness of $[X]$ is an easy exercise.

We call $[X]$ the fundamental homology class of $X$.

Resolution of singularities is not necessary for the proof of Theorem 1.1, cf. [15] and [6] for other two completely different proofs. 
Given a Zariski closed $k$-dimensional subvariety $Y$ of $X$ (for $X$ as in Theorem 1.1), we call the element $H_{k}\left(i_{Y}\right)([Y])$ of $H_{k}(X, \mathbb{Z} / 2)$, where $i_{Y}: Y \hookrightarrow X$ is the inclusion map, the homology class of $X$ represented by $Y$. Denote by

$$
H_{k}^{\text {alg }}(X, \mathbb{Z} / 2)
$$

the subgroup of $H_{k}(X, \mathbb{Z} / 2)$ generated by all homology classes of $X$ represented by Zariski closed $k$-dimensional subvarieties of $X$ and set

$$
H_{*}^{\mathrm{alg}}(X, \mathbb{Z} / 2)=\bigoplus_{k \geq 0} H_{k}^{\mathrm{alg}}(X, \mathbb{Z} / 2) .
$$

If $X$ is nonsingular, we set

$$
\begin{aligned}
H_{\text {alg }}^{k}(X, \mathbb{Z} / 2) & =D^{-1}\left(H_{d-k}^{\text {alg }}(X, \mathbb{Z} / 2)\right), \\
H_{\text {alg }}^{*}(X, \mathbb{Z} / 2) & =\bigoplus_{k \geq 0} H_{\text {alg }}^{k}(X, \mathbb{Z} / 2),
\end{aligned}
$$

where $D: H^{*}(X, \mathbb{Z} / 2) \longrightarrow H_{*}(X, \mathbb{Z} / 2)$ is the Poincaré duality isomorphism, $D(u)=$ $u \cap[X]$ for $u$ in $H^{*}(X, \mathbb{Z} / 2)$.

EXAMPLE 1.2 . It is obvious that $H_{*}^{\text {alg }}\left(\mathbb{P}^{n}(\mathbb{R}), \mathbb{Z} / 2\right)=H_{*}\left(\mathbb{P}^{n}(\mathbb{R}), \mathbb{Z} / 2\right)$. More generally, let $\mathbb{G}_{p, q}(\mathbb{R})$ be the Grassmann variety of $q$-dimensional vector subspaces of $\mathbb{R}^{p}$. A standard argument involving the Schubert cycles on $\mathbb{G}_{p, q}(\mathbb{R})$ shows that $H_{*}^{\text {alg }}\left(\mathbb{G}_{p, q}(\mathbb{R}), \mathbb{Z} / 2\right)=$ $H_{*}\left(\mathbb{G}_{p, q}(\mathbb{R}), \mathbb{Z} / 2\right)$ (cf. [6, Proposition 11.3.3]).

Basic properties of $H_{k}^{\text {alg }}$ and $H_{\text {alg }}^{*}$ are stated in the following two theorems, which are consequences of $[15$, Section 5].

THEOREM 1.3. For every regular map $f: X \rightarrow Y$ between compact real algebraic varieties

(i) $H_{*}(f)\left(H_{*}^{\text {alg }}(X, \mathbb{Z} / 2)\right) \subseteq H_{*}^{\text {alg }}(Y, \mathbb{Z} / 2)$,

(ii) $H^{*}(f)\left(H_{\text {alg }}^{*}(Y, \mathbb{Z} / 2)\right) \subseteq H_{\text {alg }}^{*}(X, \mathbb{Z} / 2)$, provided that $X$ and $Y$ are nonsingular.

THEOREM 1.4. For every compact nonsingular real algebraic variety $X$ the group $H_{\mathrm{alg}}^{*}(X, \mathbb{Z} / 2)$ is a subring of the cohomology ring $H^{*}(X, \mathbb{Z} / 2)$.

We now proceed to discuss properties of the Stiefel-Whitney classes of algebraic vector bundles.

An algebraic vector bundle on a real algebraic variety $X$ is a triple $\xi=(E, \pi, X)$, where $E$ is a real algebraic variety, $\pi: E \longrightarrow X$ is a regular map, every fiber $E_{x}=\pi^{-1}(x)$ is a real vector space for $x$ in $X$, and the usual local triviality property is satisfied. It is known that every algebraic vector bundle $\xi$ on $X$ is generated by global regular sections (cf. [24] for a simple proof; the reader should keep in mind that algebraic vector bundles considered here are often called strongly algebraic vector bundles in the literature $[2,3,4,6,13,24])$. As a consequence, if $\xi$ is of constant rank $q$, then there exists a regular map $f: X \longrightarrow \mathbb{G}_{p, q}(\mathbb{R})$, for some $p>q$, such that $\xi$ is algebraically isomorphic to the pullback vector bundle $f^{*} \gamma_{p, q}$ of the universal vector bundle $\gamma_{p, q}$ on $\mathbb{G}_{p, q}(\mathbb{R})[6$, Theorem 12.1.7].

THEOREM 1.5. If $\xi$ is an algebraic vector bundle on a compact nonsingular real algebraic variety $X$, then the total Stiefel-Whitney class $w(\xi)$ of $\xi$ belongs to $H_{\mathrm{alg}}^{*}(X, \mathbb{Z} / 2)$.

Proof. We may assume that $\xi$ has constant rank $q$ and is isomorphic to $f^{*} \gamma_{p, q}$ for some regular map $f: X \longrightarrow \mathbb{G}_{p, q}(\mathbb{R})$. Then $w(\xi)=w\left(f^{*} \gamma_{p, q}\right)=H^{*}(f)\left(w\left(\gamma_{p, q}\right)\right)$ and hence the conclusion follows in view of Example 1.2 and Theorem 1.3 (ii). 
A different proof, which contains additional useful information, can be found in [15].

Corollary 1.6. The total Stiefel-Whitney class $w(X)$ of $X$ is in $H_{\mathrm{alg}}^{*}(X, \mathbb{Z} / 2)$ for every compact nonsingular real algebraic variety $X$. In particular, $H_{\mathrm{alg}}^{1}(X, \mathbb{Z} / 2) \neq 0$ if $X$ is nonorientable as a $\mathcal{C}^{\infty}$ manifold.

Proof. Since $w(X)=w(T X)$, where $T X$ is the tangent bundle to $X$, and since $X$ is orientable if and only if $w_{1}(X)=0$, the conclusions follow from Theorem 1.5.

The next result is very useful in the study of the groups $H_{\mathrm{alg}}^{k}$ for $k=1,2$.

THEOREM 1.7. Let $X$ be a compact nonsingular real algebraic variety and let $u$ be an element of $H_{\mathrm{alg}}^{k}(X, \mathbb{Z} / 2)$.

(i) If $k=1$, then there exists a unique (up to algebraic isomorphism) algebraic line bundle $\xi$ on $X$ with $w_{1}(\xi)=u$.

(ii) If $k=2$, then there exists an algebraic vector bundle $\xi$ on $X$ with $w_{1}(\xi)=0$ and $w_{2}(\xi)=u$.

A proof of (i) is given in [6, Proposition 12.4.5, Theorem 12.4.8] (cf. also [15, Section 5]), while (ii), which is considerably harder, follows from [15, Section 5] and Grothendieck's formula [18, Example 15.3.6].

2. Rational, algebraic, and homological equivalence of real algebraic cycles. Let $V$ be a reduced quasiprojective scheme over $\mathbb{R}$. The group $Z_{k}(V)$ of algebraic $k$-cycles on $V$ is the free Abelian group generated by the prime $k$-cycles, that is, the closed integral $k$-dimensional subschemes of $V$. We let $P_{k}(V)$ denote the subgroup of $Z_{k}(V)$ of all $k$-cycles rationally equivalent to 0 (cf. [18]). As usual, two cycles $z_{1}$ and $z_{2}$ in $Z_{k}(V)$ are said to be rationally equivalent if $z_{1}-z_{2}$ is in $P_{k}(V)$. The quotient group $\mathrm{CH}_{k}(V)=Z_{k}(V) / P_{k}(V)$ is called the Chow group of $V$. Of course, if $V$ is nonsingular of pure dimension $d$, then $Z_{d-1}(V)$ is the group of Weil divisors, $P_{d-1}(V)$ is the subgroup of principal divisors (for divisors rational equivalence coincides with linear equivalence), and hence $\mathrm{CH}_{d-1}(V)$ is the divisor class group of $V$.

Denote by $V(\mathbb{R})$ the set of $\mathbb{R}$-rational points of $V$. If $V(\mathbb{R})$ is dense in $V$, then we regard $V(\mathbb{R})$ as a real algebraic variety with structure sheaf $\mathcal{R}_{V(\mathbb{R})}=\mathcal{O}_{V} \mid V(\mathbb{R})$, where $\mathcal{O}_{V}$ is the structure sheaf of $V$ (note that every real algebraic variety is biregularly isomorphic to $V(\mathbb{R})$ for some $V$ as above).

Assume now that $V(\mathbb{R})$ is dense in $V$, and $V(\mathbb{R})$ is compact (in the Euclidean topology). There exists a unique group homomorphism

$$
\mathrm{cl}_{k}: Z_{k}(V) \longrightarrow H_{k}(V(\mathbb{R}), \mathbb{Z} / 2)
$$

such that for every prime $k$-cycle $W$ on $V$ with $W(\mathbb{R})$ dense in $W$ the homology class $\operatorname{cl}_{k}(W)$ is represented by the $k$-dimensional subvariety $W(\mathbb{R})$ of $V(\mathbb{R})$, and $\operatorname{cl}_{k}(W)=0$ for all other prime $k$-cycles $W$ on $V$. By construction,

$$
\operatorname{cl}_{k}\left(Z_{k}(V)\right)=H_{k}^{\text {alg }}(V(\mathbb{R}), \mathbb{Z} / 2) .
$$

Two cycles $z_{1}$ and $z_{2}$ in $Z_{k}(V)$ are said to be homologically equivalent if $\operatorname{cl}_{k}\left(z_{1}\right)=\operatorname{cl}_{k}\left(z_{2}\right)$. It is known that

$$
P_{k}(V) \subseteq \operatorname{ker} \mathrm{cl}_{k}
$$

in other words, two rationally equivalent cycles are also homologically equivalent [15, 5.13] (this is intuitively clear since if $z_{1}$ and $z_{2}$ are rationally equivalent, then there exists 
an algebraic family $w_{t}$ of $k$-cycles on $V$, parametrized by the closed points $t$ of $\mathbb{P}_{\mathbb{R}}^{n}$, such that $z_{1}=w_{t_{1}}$ and $z_{2}=w_{t_{2}}$ for some $t_{1}$ and $t_{2}$ in $\left.\mathbb{P}_{\mathbb{R}}^{n}(\mathbb{R})=\mathbb{P}^{n}(\mathbb{R})\right)$. The homomorphism $\mathrm{CH}_{k}(V) \longrightarrow H_{k}(V(\mathbb{R}), \mathbb{Z} / 2)$ induced by $\mathrm{cl}_{k}$ has nice functorial properties, which in fact imply Theorems 1.3 and 1.4 (cf. [15] for details).

A cycle $z$ in $Z_{k}(V)$ is said to be thin if for every prime $k$-cycle $W$ that appears in $z$ with a nonzero coefficient the set $W(\mathbb{R})$ is not dense in $W$. By definition of $\mathrm{cl}_{k}$, the group $Z_{k}^{\text {th }}(V)$ of all thin cycles on $V$ satisfies

$$
Z_{k}^{\mathrm{th}}(V) \subseteq \operatorname{ker~cl}_{k}
$$

The main result of $[25]$ is the following

THEOREM 2.1. Let $X$ be a nonsingular quasiprojective scheme over $\mathbb{R}$ of pure dimension d. If $V(\mathbb{R})$ is dense in $V$ and $V(\mathbb{R})$ is compact, then

$$
\operatorname{ker~cl}_{k}=P_{k}(V)+Z_{k}^{\mathrm{th}}(V)
$$

for every integer $k$ satisfying $0 \leq k \leq d-1$.

Observe that Theorem 2.1 allows us to give a purely algebraic geometric description of $H_{k}^{\text {alg }}(V(\mathbb{R}), \mathbb{Z} / 2)$ as the quotient group $Z_{k}(V) /\left(P_{k}(V)+Z_{k}^{\text {th }}(V)\right)$.

Theorem 2.1 means precisely that an algebraic $k$-cycle on $V$ is homologically equivalent to 0 if an only if it is rationally equivalent to a thin cycle. For divisors this can be strengthened as follows (cf. [17]).

TheOREM 2.2. Let $V$ be as in Theorem 2.1. A divisor on $V$ is homologically equivalent to 0 if and only if it is linearly equivalent to a divisor whose support is disjoint from $V(\mathbb{R})$.

Each of the two theorems above is a generalization of a classical result due to Witt [40].

We conclude this section with a brief discussion of algebraic equivalence. Let $Q_{k}(V)$ denote the subgroup of $Z_{k}(V)$ of all $k$-cycles algebraically equivalent to 0 (cf. [18]). Two cycles $z_{1}$ and $z_{2}$ in $Z_{k}(V)$ are said to be algebraically equivalent if $z_{1}-z_{2}$ is in $Q_{k}(V)$. Roughly, this means that there exists an algebraic family $w_{t}$ of $k$-cycles on $V$, parametrized by the closed points $t$ of an irreducible nonsingular quasiprojective scheme $T$ over $\mathbb{R}$, such that $z_{1}=w_{t_{1}}$ and $z_{2}=w_{t_{2}}$ for some $t_{1}$ and $t_{2}$ in $T(\mathbb{R})$. Since $t_{1}$ and $t_{2}$ can be in different connected components of $T(\mathbb{R})$, there is no obvious reason why $z_{1}$ and $z_{2}$ should be homologically equivalent and, in general, they are not. As demonstrated by many examples in [28], $\operatorname{cl}_{k}\left(Q_{k}(V)\right) \neq 0$ can occur. The next result also comes from [28].

TheOrem 2.3. Let $V$ be as in Theorem 2.1. If $\operatorname{dim}_{\mathbb{Z} / 2} \operatorname{cl}_{k}\left(Q_{k}(V)\right) \geq \ell$, then

$$
\operatorname{dim}_{\mathbb{Z} / 2} H_{d-k}(V(\mathbb{R}), \mathbb{Z} / 2) / H_{d-k}^{\mathrm{alg}}(V(\mathbb{R}), \mathbb{Z} / 2) \geq \ell \text {. }
$$

3. Algebraic models of $\mathcal{C}^{\infty}$ manifolds. Given a $\mathcal{C}^{\infty}$ manifold $M$, we call a nonsingular real algebraic variety $X$ diffeomorphic to $M$ an algebraic model of $M$. By Tognoli's theorem (cf. [39] or [6, Theorem 14.1.10]), every closed $\mathcal{C}^{\infty}$ manifold $M$ has an algebraic model.

In this section we are concerned with algebraic models of closed $\mathcal{C}^{\infty}$ manifolds with some groups $H_{\mathrm{alg}}^{k}$ given in advance. We begin with an example to illustrate the occuring phenomena (cf. [27] and [6, Theorem 11.3.6]). 
EXAmple 3.1. Denote by $S^{p}$ the unit sphere in $\mathbb{R}^{p+1}$. Let $M$ be a $\mathcal{C}^{\infty}$ manifold which is the connected sum of $s$ copies of $S^{n} \times S^{k}, s \geq 1, n \geq 1, k \geq 1$ (note that, in particular, every closed connected orientable $\mathcal{C}^{\infty}$ surface of positive genus $g$ can be obtained in this fashion with $s=g$ and $n=k=1$ ). Below we construct an algebraic model $X$ of $M$ satisfying $H_{\mathrm{alg}}^{k}(X, \mathbb{Z} / 2) \neq H^{k}(X, \mathbb{Z} / 2)$.

Let $C=\left\{\left(x_{1}, \ldots, x_{n+1}\right) \in \mathbb{R}^{n+1} \mid x_{1}^{4}-4 x_{1}^{2}+1+x_{2}^{2}+\ldots+x_{n+1}^{2}=0\right\}$. Clearly, $C$ is a nonsingular irreducible algebraic subset of $\mathbb{R}^{n+1}$ with two connected components $C_{1}$ and $C_{2}$, each of which is diffeomorphic to $S^{n}$. Set $W=C \times S^{k+1}$. Choose a compact $\mathcal{C}^{\infty}$ submanifold $B$ of $S^{k+1}$ whose boundary $\partial B$ has $s$ connected components, each of which is diffeomorphic to $S^{k}$. Choose a point $y$ in $\partial B$. By joining the connected components of $C_{1} \times \partial B$ with "tubes" in $W$ we can construct a compact $\mathcal{C}^{\infty}$ submanifold $V$ of $W$ with boundary $\partial V=N$ diffeomorphic to $M$ and containing $C_{1} \times\{y\}$. Since $N$ bounds in $W$, there exists a $\mathcal{C}^{\infty}$ function $f: W \longrightarrow \mathbb{R}$ such that $0 \in \mathbb{R}$ is a regular value of $f$ and $N=f^{-1}(0)$. The classical Weierstrass approximation theorem allows us to find a polynomial function $p: W \longrightarrow \mathbb{R}$ close to $f$ in the $\mathcal{C}^{\infty}$ topology. If $p$ is sufficiently close to $f$, then $X=p^{-1}(0)$ is a nonsingular algebraic subset of $W$ and there exists a $\mathcal{C}^{\infty}$ diffeomorphism $\varphi: W \longrightarrow W$, close in the $\mathcal{C}^{\infty}$ topology to the identity map of $W$, with $\varphi(N)=X$ (in particular, $X$ is an algebraic model of $M$ ). Furthermore, the $\mathcal{C}^{\infty}$ submanifold $D=\varphi\left(C_{1} \times\{y\}\right)$ of $X$ is mapped diffeomorphically onto $C_{1}$ by the canonical projection $\pi: W \longrightarrow C$. Let $u$ be the homology class in $H_{n}(X, \mathbb{Z} / 2)$ represented by $D$. Then $H_{n}(\pi)(u)$ is the homology class in $H_{n}(C, \mathbb{Z} / 2)$ represented by $C_{1}$, and hence $H_{n}(\pi)(u)$ is not in $H_{n}^{\text {alg }}(C, \mathbb{Z} / 2)(C$ is irreducible of dimension $n)$. Thus, in view of Theorem $1.3(\mathrm{i}), u$ is not in $H_{n}^{\text {alg }}(X, \mathbb{Z} / 2)$, which implies $H_{\text {alg }}^{k}(X, \mathbb{Z} / 2) \neq H^{k}(X, \mathbb{Z} / 2)$.

The next two general results come from [9].

THEOREM 3.2. Let $M$ be a closed connected $\mathcal{C}^{\infty}$ manifold of dimension at least 3 and let $G$ be a subgroup of $H^{1}(M, \mathbb{Z} / 2)$. Then the following conditions are equivalent:

(a) There exist an algebraic model $X$ of $M$ and a diffeomorphism $\varphi: X \longrightarrow M$ such that $H^{1}(\varphi)(G)=H_{\text {alg }}^{1}(X, \mathbb{Z} / 2)$;

(b) $w_{1}(M) \in G$.

By Corollary 1.6, (a) implies (b), while the proof of $(b) \Rightarrow(a)$ is rather involved.

For surfaces only a somewhat weaker result is known. Given a compact nonsingular real algebraic surface $X$, we set

$$
\begin{gathered}
\beta(X)=\operatorname{dim}_{\mathbb{Z} / 2} H_{\text {alg }}^{1}(X, \mathbb{Z} / 2), \\
\delta(X)=\operatorname{dim}_{\mathbb{Z} / 2}\left\{v \in H_{\text {alg }}^{1}(X, \mathbb{Z} / 2) \mid v \cup v=0\right\} .
\end{gathered}
$$

If $X$ is connected and orientable (resp. nonorientable of odd topological genus), then $\beta(X)=\delta(X)$ (resp. $\beta(X)=\delta(X)+1)$. For $X$ connected nonorientable of even topological genus one has either $\beta(X)=\delta(X)$ or $\beta(X)=\delta(X)+1$. These statements are consequences of elementary topological arguments and $w_{1}(X) \in H_{\mathrm{alg}}^{1}(X, \mathbb{Z} / 2)$. The next result shows that all topologically possible cases can be realized algebraically.

THEOREM 3.3.

(i) Let $M$ be a closed connected $\mathcal{C}^{\infty}$ surface of genus $g$ and let $\beta$ be an integer satisfying

$$
\begin{array}{ll}
0 \leq \beta \leq 2 g & \text { for } M \text { orientable } \\
1 \leq \beta \leq g & \text { for } M \text { nonorientable. }
\end{array}
$$


Then there exists an algebraic model $X_{\beta}$ of $M$ with $\beta\left(X_{\beta}\right)=\beta$.

(ii) Let $M$ be a closed connected nonorientable $\mathcal{C}^{\infty}$ surface of even genus $g$ and let $\beta$ and $\delta$ be integers satisfying either

$$
\beta=\delta+1, \quad 2 \leq \beta \leq g
$$

or

$$
\beta=\delta, \quad 1 \leq \beta \leq g-1 .
$$

Then there exists an algebraic model $X_{\beta, \delta}$ of $M$ such that $\beta\left(X_{\beta, \delta}\right)=\beta$ and $\delta\left(X_{\beta, \delta}\right)=\delta$.

We shall now deal with $H_{\text {alg }}^{2}$. Given a topological space $M$, denote by $W^{2}(M)$ the subset of $H^{2}(M, \mathbb{Z} / 2)$ consisting of all elements of the form $w_{2}(\xi)$ for some topological real vector bundle $\xi$ on $M$. One easily checks that if $M$ is paracompact, then every element of $W^{2}(M)$ is of the form $w_{2}(\xi)$ with $w_{1}(\xi)=0$, and hence $W^{2}(M)$ is a subgroup of $H^{2}(M, \mathbb{Z} / 2)$. In [11] one finds the following

THEOREM 3.4. Let $M$ be a closed connected orientable $\mathcal{C}^{\infty}$ manifold of dimension at least 5 and let $G$ be a subgroup of $H^{2}(M, \mathbb{Z} / 2)$. Then the following conditions are equivalent:

(a) There exist an algebraic model $X$ of $M$ and a diffeomorphism $\varphi: X \longrightarrow M$ such that $H^{2}(\varphi)(G)=H_{\text {alg }}^{2}(X, \mathbb{Z} / 2)$;

(b) $w_{2}(M) \in G$ and $G \subseteq W^{2}(M)$.

We note that $(\mathrm{a}) \Rightarrow(\mathrm{b})$ follows from Corollary 1.6 and Theorem 1.7 (ii), and hence the difficulty lies in showing $(b) \Rightarrow(a)$.

Theorem 3.4 is nicely complemented by the following result of [37], in which $|H|$ denotes the order of a group $H$.

THEOREM 3.5. Given an integer $n \geq 6$, there exists a closed connected orientable $\mathcal{C}^{\infty}$ manifold $M$ of dimension $n$ such that $H^{2}(M, \mathbb{Z} / 2) \neq 0$ and for every algebraic model $X$ of $M$ one has

$$
\left|H_{\text {alg }}^{2}(X, \mathbb{Z} / 2)\right| \leq \frac{1}{2}\left|H^{2}(X, \mathbb{Z} / 2)\right|
$$

It follows from Theorem 3.4 that $W^{2}(M) \neq H^{2}(M, \mathbb{Z} / 2)$ for $M$ as in Theorem 3.5.

In dimensions less than 6 the situation is quite different.

TheOREM 3.6. For every closed $\mathcal{C}^{\infty}$ manifold $M$ of dimension less than 6 there exists an algebraic model $X$ of $M$ satisfying $H_{\mathrm{alg}}^{*}(X, \mathbb{Z} / 2)=H^{*}(X, \mathbb{Z} / 2)$.

Pr o of. By Thom's theorem [38, Theorem II.26], there exist closed $\mathcal{C}^{\infty}$ submanifolds $M_{1}, \ldots, M_{k}$ of $M$ whose homology classes generate $H_{*}(M, \mathbb{Z} / 2)$. We may assume that the $M_{i}$ are in general position. Then it follows from [2, Theorem 4] that there exist an algebraic model $X$ of $M$ and a diffeomorphism $h: M \longrightarrow X$ such that $h\left(M_{i}\right)$ is a Zariski closed nonsingular subvariety of $X$ for $i=1, \ldots, k$. This implies the conclusion.

We close with a remark that no general result analogous to Theorems 3.2 and 3.4 is known for $H_{\text {alg }}^{k}, k \geq 3$. 
4. The group $H_{\mathrm{alg}}^{1}$ of the underlying real algebraic structure of a complex projective variety. Every complex projective variety $V$ of dimension $n$ can be regarded as a real algebraic variety of dimension $2 n$, and as such will be denoted by $V_{\mathbb{R}}$. Assume that $V$ is nonsingular and irreducible. Due to [19, 22], a method for computing the group $H_{\text {alg }}^{1}\left(V_{\mathbb{R}}, \mathbb{Z} / 2\right)$ is well understood. We shall now present the main results, concentrating on comparison between the real algebraic geometry invariant

$$
d(V)=\operatorname{dim}_{\mathbb{Z} / 2} H_{\text {alg }}^{1}\left(V_{\mathbb{R}}, \mathbb{Z} / 2\right)
$$

and the topological invariants

$$
h(V)=\operatorname{dim}_{\mathbb{Z} / 2} H^{1}(V, \mathbb{Z} / 2),
$$

$$
b_{1}(V)=\text { the first Betti number of } V=\operatorname{dim}_{\mathbb{Z} / 2}\left(H^{1}(V, \mathbb{Z}) \otimes_{\mathbb{Z}} \mathbb{Z} / 2\right) .
$$

Recall first that to $V$ we can attach functorially a complex Abelian variety, the Albanese variety $\operatorname{Alb}(V)$ of $V$, and a regular map $\alpha: V \longrightarrow \operatorname{Alb}(V)$, the Albanese map. For curves ( $\operatorname{dim} V=1) \operatorname{Alb}(V)$ is simply the Jacobian variety of $V$. The dimension of $\operatorname{Alb}(V)$ is equal to $\frac{1}{2} b_{1}(V)$ (it is well known that $b_{1}(V)$ is even).

The following result of [19] plays an important role.

TheOREM 4.1. The Albanese map $\alpha: V \longrightarrow \mathrm{Alb}(V)$ induces an isomorphism

$$
H^{1}(\alpha): H_{\text {alg }}^{1}\left((\operatorname{Alb}(V))_{\mathbb{R}}, \mathbb{Z} / 2\right) \longrightarrow H_{\text {alg }}^{1}\left(V_{\mathbb{R}}, \mathbb{Z} / 2\right) .
$$

The computation of $H_{\mathrm{alg}}^{1}\left(V_{\mathbb{R}}, \mathbb{Z} / 2\right)$ is therefore reduced to the case of Abelian varieties, which was earlier investigated in [22].

For a complex Abelian variety $A$ the invariant $d(A)$ can be computed in terms of a period matrix of $A$ (of course, $b_{1}(A)=h(A)=2 \operatorname{dim} A$ ). We shall describe this method in the case of 1-dimensional complex Abelian varieties, that is, elliptic curves.

Let $E_{\tau}=\mathbb{C} /(\mathbb{Z}+\mathbb{Z} \tau)$ be a complex elliptic curve, $\tau \in \mathbb{C} \backslash \mathbb{R}$. We shall express $d\left(E_{\tau}\right)$ as an explicitly computable function of $\tau$. Let $S_{\tau}$ and $T_{\tau}$ be the subgroups of $\mathbb{Z}^{2}$ defined as follows:

$$
\begin{gathered}
S_{\tau}=\left\{\left.(m, n) \in \mathbb{Z}^{2}|m+n| \tau\right|^{2} \in 2 \operatorname{Re} \tau \mathbb{Z}\right\}, \\
T_{\tau}=S_{\tau} \cap 2 \mathbb{Z}^{2} .
\end{gathered}
$$

THEOREM 4.2. With the notation as above, $d\left(E_{\tau}\right)=\operatorname{dim}_{\mathbb{Z} / 2} S_{\tau} / T_{\tau}$.

This result is a consequence of $[19,22]$. It allows us to deduce several interesting corollaries (cf. [12, 22]).

EXAMPLE 4.3.

(i) If $\tau=\alpha \sqrt{-1}$, where $\alpha \in \mathbb{R} \backslash\{0\}$, then

$$
d\left(E_{\tau}\right)= \begin{cases}1 & \text { if } \alpha^{2} \in \mathbb{Q} \\ 0 & \text { if } \alpha^{2} \notin \mathbb{Q} .\end{cases}
$$

(ii) If $\tau=\frac{1}{2}(1+\sqrt{-d})$, where $d$ is a positive integer satisfying $d \equiv 3(\bmod 4)$, then $d\left(E_{\tau}\right)=2$.

Recall that a complex elliptic curve $E$ is said to have complex multiplication if the ring $\operatorname{End}(E)$ of endomorphisms of $E$ is not isomorphic to $\mathbb{Z}$. In such a case $\operatorname{End}(E) \cong$ 
$\mathbb{Z}+f \mathcal{O}(-d)$, where $d$ and $f$ are positive integers, $d$ is square free, and $\mathcal{O}(-d)$ is the ring of integers in the quadratic field $\mathbb{Q}(\sqrt{-d})$. The integer

$$
\delta(E)=\left\{\begin{array}{lll}
-f^{2} d & \text { if } d \equiv 3(\bmod 4) \\
-4 f^{2} d & \text { if } d \neq \equiv 3(\bmod 4)
\end{array}\right.
$$

is called the discriminant of $E$.

Corollary 4.4. Let E be a complex elliptic curve with complex multiplication. Then

$$
d(E)= \begin{cases}1 & \text { if } \delta(E) \text { is even } \\ 2 & \text { if } \delta(E) \text { is odd } .\end{cases}
$$

Proof. Let $F=\mathbb{C} /(\mathbb{Z}+\tau \mathbb{Z})$, where $\tau=\frac{1}{2} \sqrt{\delta(E)}$ if $\delta(E)$ is even, and $\tau=\frac{1}{2}(1+\sqrt{\delta(E)})$ if $\delta(E)$ is odd. By Example $4.3(\mathrm{i})$, one has $d(F)=1$ if $\delta(E)$ is even. If $\delta(E)$ is odd, then $-\delta(E) \equiv 3(\bmod 4)$ and hence $d(F)=2$ in view of Example 4.3 (ii). Since $\delta(E)=\delta(F)$, according to [23], the real algebraic varieties $E_{\mathbb{R}}$ and $F_{\mathbb{R}}$ are isomorphic. The corollary follows.

A more precise description of the complex elliptic curves $E$ satisfying $H_{\mathrm{alg}}^{1}\left(E_{\mathbb{R}}, \mathbb{Z} / 2\right)=$ $H^{1}\left(E_{\mathbb{R}}, \mathbb{Z} / 2\right.$ ) (equivalently, $d(E)=2$ ) is provided by the next statement.

THEOREM 4.5. Given a complex elliptic curve E, the following conditions are equivalent:

(a) $H_{\text {alg }}^{1}\left(E_{\mathbb{R}}, \mathbb{Z} / 2\right)=H^{1}\left(E_{\mathbb{R}}, \mathbb{Z} / 2\right)$;

(b) E has complex multiplication and $\delta(E)$ is odd;

(c) $E_{\mathbb{R}}$ is biregularly isomorphic to the product of real algebraic curves.

The implication (a) $\Rightarrow$ (b) follows from Theorem 4.2 and Corollary 4.4 , while (b) $\Rightarrow$ (c) is proved in [7]. The implication (c) $\Rightarrow$ (a) is trivial.

Some results on elliptic curves generalize to higher dimensional complex Abelian varieties. First recall that a simple complex Abelian variety $A$ is said to have complex multiplication if the rank of the $\operatorname{ring} \operatorname{End}(A)$ is equal to $2 \operatorname{dim} A$. An arbitrary complex Abelian variety is said to have complex multiplication if it is isogenous to the product of simple Abelian varieties with complex multiplication.

The next result comes from [19] and is partially based on [22].

TheOrem 4.6. If $A$ is a complex Abelian variety satisfying

$$
H_{\text {alg }}^{1}\left(A_{\mathbb{R}}, \mathbb{Z} / 2\right)=H^{1}\left(A_{\mathbb{R}}, \mathbb{Z} / 2\right),
$$

then A has complex multiplication.

In particular, the set of isomorphism classes of complex Abelian varieties $A$ with $H_{\text {alg }}^{1}\left(A_{\mathbb{R}}, \mathbb{Z} / 2\right)=H^{1}\left(A_{\mathbb{R}}, \mathbb{Z} / 2\right)$ is countable.

Let us return to Theorem 4.1. Observe that the homomorphism

$$
H^{1}(\alpha): H^{1}(\operatorname{Alb}(V), \mathbb{Z} / 2) \longrightarrow H^{1}(V, \mathbb{Z} / 2),
$$

induced by the Albanese map $\alpha: V \longrightarrow \operatorname{Alb}(V)$, need not be surjective. In fact, it is surjective if and only if $H^{2}(V, \mathbb{Z})$ has no 2-torsion. Therefore the topology of $V$ imposes strong restrictions on the existence of homology classes in codimension 1 represented by real algebraic hypersurfaces of $V_{\mathbb{R}}$. Theorem 4.1 implies the next two corollaries, in which $V$ is a nonsingular irreducible complex projective variety. 
COROLlary 4.7. The group $H_{\mathrm{alg}}^{1}\left(V_{\mathbb{R}}, \mathbb{Z} / 2\right)$ is contained in the image of the canonical monomorphism

$$
H^{1}(V, \mathbb{Z}) \otimes_{\mathbb{Z}} \mathbb{Z} / 2 \longrightarrow H^{1}(V, \mathbb{Z} / 2) .
$$

In particular, $d(V) \leq b_{1}(V)$.

COROLlary 4.8. If the group $H^{2}(V, \mathbb{Z})$ has 2-torsion, then

$$
H_{\text {alg }}^{1}\left(V_{\mathbb{R}}, \mathbb{Z} / 2\right) \neq H^{1}\left(V_{\mathbb{R}}, \mathbb{Z} / 2\right) .
$$

As a special case one obtains the following

EXAMPLE 4.9. Each complex Enriques surface $F$ has $b_{1}(F)=0$ and hence $H_{\text {alg }}^{1}\left(F_{\mathbb{R}}, \mathbb{Z} / 2\right)=0$. On the other hand, $H^{1}(F, \mathbb{Z} / 2)=\mathbb{Z} / 2$.

More generally, a construction of complex algebraic varieties $V$ with prescribed $\operatorname{dim} V \geq 2, d(V), b_{1}(V)$, and $h(V)$ is given in [19].

THEOREM 4.10. Given nonnegative integers $n, a, g, h, n \geq 2$, the following conditions are equivalent:

(a) There exists a nonsingular irreducible complex projective variety $V$ such that $\operatorname{dim} V=n, d(V)=a, b_{1}(V)=2 g$, and $h(V)=h$;

(b) $0 \leq a \leq 2 g \leq h$.

Sketch of proof. By Corollary 4.7, (a) implies (b).

Assume that (b) holds. We know that there exists a complex elliptic curve $E_{j}$ with $d\left(E_{j}\right)=j$ for $j=0,1,2$ (Example 4.3). Let $F$ be a complex Enriques surface (cf. Example 4.9). By taking the product of an appropriate number of $E_{0}, E_{1}, E_{2}$, and $F$, we can construct $V^{\prime}$ satisfying $d\left(V^{\prime}\right)=a, b_{1}\left(V^{\prime}\right)=2 g$, and $h\left(V^{\prime}\right)=h$. If $\operatorname{dim} V^{\prime} \leq n$, then (a) holds for $V=V^{\prime} \times \mathbb{P}^{\ell}(\mathbb{C})$, where $\ell=n-\operatorname{dim} V^{\prime}$. If $\operatorname{dim} V^{\prime}>n$, then $V$ satisfying (a) can be obtained by embedding $V^{\prime}$ in $\mathbb{P}^{N}(\mathbb{C})$ and intersecting it with $\operatorname{dim} V^{\prime}-n$ hyperplanes (this can be deduced from the Lefschetz theorem on hyperplane sections).

No result analogous to Theorem 4.10 is known for complex curves. We can only formulate the following

Conjecture 4.11. Let $g$ and $a$ be integers, $0 \leq a \leq 2 g$. Then there exists a nonsingular irreducible complex projective curve $V$ of genus $g$ such that $d(V)=a$ (of course, $\left.b_{1}(V)=h(V)=2 g\right)$.

A discussion of some special cases of the conjecture is given in [12].

Observe that the equality $H_{\text {alo }}^{1}\left(V_{\mathbb{R}}, \mathbb{Z} / 2\right)=H^{1}\left(V_{\mathbb{R}}, \mathbb{Z} / 2\right)$ imposes very severe restrictions on the curve $V$. Indeed, by Theorems 4.1 and 4.6 , the Jacobian variety of each such curve $V$ has complex multiplication. It follows from Torelli's theorem that the set $\mathcal{M}_{\mathrm{CM}}^{g}$ of isomorphism classes of nonsingular irreducible complex projective curves of genus $g$ whose Jacobians have complex multiplication is at most countable. Let us mention that it is an open problem whether or not the set $\mathcal{M}_{\mathrm{CM}}^{g}$ is infinite for all large $g$.

In particular, we obtain the following

COROLlARY 4.12. Up to isomorphism, there exist at most countably many nonsingular irreducible complex projective curves $V$ satisfying $H_{\text {alg }}^{1}\left(V_{\mathbb{R}}, \mathbb{Z} / 2\right)=H^{1}\left(V_{\mathbb{R}}, \mathbb{Z} / 2\right)$. 
5. Applications. In this section we briefly describe applications of the groups $H_{\text {alg }}^{k}$ to five different types of problems.

A. Regular maps into the unit circle

Let $X$ be a compact nonsingular real algebraic variety and let $\mathcal{R}\left(X, S^{1}\right)$ denote the set of all regular maps from $X$ into the unit circle $S^{1}=\left\{(x, y) \in \mathbb{R}^{2} \mid x^{2}+y^{2}=1\right\}$. We regard $\mathcal{R}\left(X, S^{1}\right)$ as a subset of the space $\mathcal{C}^{\infty}\left(X, S^{1}\right)$ of all $\mathcal{C}^{\infty}$ maps from $X$ into $S^{1}$, endowed with the $\mathcal{C}^{\infty}$ topology. The closure $\overline{\mathcal{R}\left(X, S^{1}\right)}$ of $\mathcal{R}\left(X, S^{1}\right)$ in $\mathcal{C}^{\infty}\left(X, S^{1}\right)$ is described in the following result (cf. [8]).

TheOREM 5.1. Given a $\mathcal{C}^{\infty}$ map $f$ in $\mathcal{C}^{\infty}\left(X, S^{1}\right)$, the following conditions are equivalent:

(a) $f \in \overline{\mathcal{R}\left(X, S^{1}\right)}$;

(b) $f$ is homotopic to a regular map from $X$ into $S^{1}$;

(c) $H^{1}(f)(\kappa) \in H_{\mathrm{alg}}^{1}(X, \mathbb{Z} / 2)$, where $\kappa$ is the generator of $H^{1}\left(S^{1}, \mathbb{Z} / 2\right) \cong \mathbb{Z} / 2$.

It is convenient to consider $S^{1}$ as a multiplicative group, $S^{1}=\{z \in \mathbb{C}|| z \mid=1\}$, which also allows us to regard $\mathcal{C}^{\infty}\left(X, S^{1}\right)$ as a group. Obviously, $\mathcal{R}\left(X, S^{1}\right)$ and $\overline{\mathcal{R}\left(X, S^{1}\right)}$ are subgroups of $\mathcal{C}^{\infty}\left(X, S^{1}\right)$. The quotient group

$$
\Gamma(X)=\mathcal{C}^{\infty}\left(X, S^{1}\right) / \overline{\mathcal{R}\left(X, S^{1}\right)}
$$

measures the size of $\overline{\mathcal{R}\left(X, S^{1}\right)}$ in $\mathcal{C}^{\infty}\left(X, S^{1}\right)$; in particular, $\mathcal{R}\left(X, S^{1}\right)$ is dense in $\mathcal{C}^{\infty}\left(X, S^{1}\right)$ if and only if $\Gamma(X)=0$. After introducing some notation, we describe $\Gamma(X)$ in terms of $H_{\text {alg }}^{1}(X, \mathbb{Z} / 2)$ in the next result (cf. [9]).

Given a topological space $M$, we let

$$
r_{M}: H^{1}(M, \mathbb{Z}) \longrightarrow H^{1}(M, \mathbb{Z} / 2)
$$

denote the reduction modulo 2 homomorphism and set

$$
A(M)=r_{M}\left(H^{1}(M, \mathbb{Z})\right) .
$$

Of course, $A(M)$ is canonically isomorphic to $H^{1}(M, \mathbb{Z}) \otimes_{\mathbb{Z}} \mathbb{Z} / 2$.

TheOREM 5.2. With the notation as above, $\Gamma(X)$ is canonically isomorphic to

$$
A(X) /\left(A(X) \cap H_{\text {alg }}^{1}(X, \mathbb{Z} / 2)\right) .
$$

Proof. Let $\lambda$ be a generator of $H^{1}\left(S^{1}, \mathbb{Z}\right) \cong \mathbb{Z}$ and let $\Phi: \mathcal{C}^{\infty}\left(X, S^{1}\right) \longrightarrow H^{1}(X, \mathbb{Z})$ be the homomorphism defined by $\Phi(f)=H^{1}(f)(\lambda)$ for all $f$ in $\mathcal{C}^{\infty}\left(X, S^{1}\right)$. The composite homomorphism $\Psi=r_{X} \circ \Phi: \mathcal{C}^{\infty}\left(X, S^{1}\right) \longrightarrow H^{1}(X, \mathbb{Z} / 2)$ does not depend on the choice of $\lambda$. Furthermore, since $\Phi$ is surjective (cf. [21]), it follows that

$$
\Psi\left(\mathcal{C}^{\infty}\left(X, S^{1}\right)\right)=A(X) .
$$

On the other hand, by Theorem 5.1 ,

$$
\Psi^{-1}\left(H_{\text {alg }}^{1}(X, \mathbb{Z} / 2)\right)=\overline{\mathcal{R}\left(X, S^{1}\right)}
$$

Hence $\Psi$ induces an isomorphism from $\Gamma(X)$ onto $A(X) /\left(A(X) \cap H_{\mathrm{alg}}^{1}(X, \mathbb{Z} / 2)\right)$.

Theorems 5.2, 3.2, and 3.3 imply the following (cf. [9])

COROllary 5.3. Let $M$ be a closed connected $\mathcal{C}^{\infty}$ manifold of dimension at least 2 and let

$$
\alpha(M)= \begin{cases}\operatorname{rank} H^{1}(M, \mathbb{Z})-1 & \text { if } M \text { is nonorientable and } w_{1}(M) \in A(M) \\ \operatorname{rank} H^{1}(M, \mathbb{Z}) & \text { otherwise. }\end{cases}
$$


Then

(i) For every algebraic model $X$ of $M$ one has $\Gamma(X) \cong(\mathbb{Z} / 2)^{s}$ for some integer $s$ satisfying $0 \leq s \leq \alpha(M)$.

(ii) For each integer $s$ satisfying $0 \leq s \leq \alpha(M)$ there exists an algebraic model $X_{s}$ of $M$ with $\Gamma\left(X_{s}\right) \cong(\mathbb{Z} / 2)^{s}$.

Using the invariant $\alpha(M)$, one immediately obtains the next two corollaries.

Corollary 5.4. Given a closed connected orientable $\mathcal{C}^{\infty}$ manifold $M$, the following conditions are equivalent:

(a) $\mathcal{R}\left(X, S^{1}\right)$ is dense in $\mathcal{C}^{\infty}\left(X, S^{1}\right)$ for every algebraic model $X$ of $M$;

(b) The first Betti number of $M$ is equal to 0 or $\operatorname{dim} M=1$.

Corollary 5.5. Given a closed connected $\mathcal{C}^{\infty}$ surface $M$, the following conditions are equivalent:

(a) $\mathcal{R}\left(X, S^{1}\right)$ is dense in $\mathcal{C}^{\infty}\left(X, S^{1}\right)$ for every algebraic model $X$ of $M$;

(b) $M$ is homeomorphic to $S^{2}\left(2\right.$-sphere) or $\mathbb{P}^{2}(\mathbb{R})$ (real projective plane) or the Klein bottle.

B. Hypersurfaces in real algebraic varieties

The following result is contained in many papers, cf. for example $[2,6,14]$.

THEOREM 5.6. Let $X$ be a compact nonsingular real algebraic variety of dimension $d$ and let $M$ be a closed $\mathcal{C}^{\infty}$ submanifold of $X$ of dimension $d-1$. Assume that the homology class represented by $M$ belongs to $H_{d-1}^{\text {alg }}(X, \mathbb{Z} / 2)$. Then there exists a $\mathcal{C}^{\infty}$ diffeomorphism $\sigma: X \longrightarrow X$, arbitrarily close in the $\mathcal{C}^{\infty}$ topology to the identity map of $X$, such that $\sigma(M)$ is a Zariski closed nonsingular subvariety of $X$.

C. Algebraicity of real analytic functions

Here we cite only one result of [14], which can be stated without introducing any new definitions.

THEOREM 5.7. Let $X$ be a compact connected nonsingular real algebraic surface. Then the following conditions are equivalent:

(a) Every real analytic function $f: X \longrightarrow \mathbb{R}$ is analytically equivalent to a regular function (that is, there exist a regular function $g: X \longrightarrow \mathbb{R}$ and an analytic diffeomorphism $\sigma: X \longrightarrow X$ such that $g=f \circ \sigma)$;

(b) $H_{\text {alg }}^{1}(X, \mathbb{Z} / 2)=H^{1}(X, \mathbb{Z} / 2)$.

The group $H_{\mathrm{alg}}^{1}$ intervenes also in the investigation of the problem of algebraicity of real analytic sets (cf. [14]).

D. Factoriality of the ring of regular functions

The easiest to state is the following (cf. [6, Proposition 12.4.12])

THEOREM 5.8. Let $X$ be a compact nonsingular irreducible real algebraic variety. The ring $\mathcal{R}(X)$ of regular functions on $X$ is a unique factorization domain if and only if $H_{\text {alg }}^{1}(X, \mathbb{Z} / 2)=0$. 
Recall that one associates with each Krull domain $A$ the divisor class group $\mathrm{Cl}(A)$ (cf. $[16$, Chapter $7, \S 1])$. The main property of $\mathrm{Cl}(A)$ is that $A$ is a unique factorization domain if and only if $\mathrm{Cl}(A)=0$. One shows that for $X$ as above, $\mathrm{Cl}(\mathcal{R}(X))$ is isomorphic to the Picard group $\operatorname{Pic}(\mathcal{R}(X))$ of all invertible $\mathcal{R}(X)$-modules, which in turn is isomorphic to $H_{\text {alg }}^{1}(X, \mathbb{Z} / 2)[6$, Sections $12.2-12.4]$.

By combining Theorem 5.8 and Theorems 3.2 and 3.3, we obtain the following

Corollary 5.9. Let $M$ be a closed connected $\mathcal{C}^{\infty}$ manifold of dimension at least 2. Then the following conditions are equivalent:

(a) There exists an algebraic model $X$ of $M$ such that $\mathcal{R}(X)$ is a unique factorization domain;

(b) $M$ is orientable.

E. K-theory of real algebraic surfaces and threefolds

Let $X$ be a real algebraic variety. It is known that the Grothendieck group $K_{0}(\mathcal{R}(X))$ of finitely generated projective $\mathcal{R}(X)$-modules is canonically isomorphic to the Grothendieck group of algebraic vector bundles on $X$ [6, Theorem 12.1.7].

Under some restrictions on $X$, the group $K_{0}(\mathcal{R}(X))$ is computed in [10]. We cite this result below.

THEOREM 5.10. Let $X$ be a compact nonsingular real algebraic variety of dimension at most 3. Then

$$
K_{0}(\mathcal{R}(X)) \cong \mathbb{Z}^{\beta_{0}(X)} \oplus(\mathbb{Z} / 4)^{\beta_{1}(X)-\delta(X)} \oplus(\mathbb{Z} / 2)^{\beta_{1}(X)+\beta_{2}(X)-\delta(X)},
$$

where

$$
\begin{gathered}
\beta_{k}(X)=\operatorname{dim}_{\mathbb{Z} / 2} H_{\text {alg }}^{k}(X, \mathbb{Z} / 2), \\
\delta(X)=\operatorname{dim}_{\mathbb{Z} / 2}\left\{v \in H_{\text {alg }}^{1}(X, \mathbb{Z} / 2) \mid v \cup v=0\right\} .
\end{gathered}
$$

Probably it is now illuminating to have another look at Theorems 3.2 and 3.3.

Let us mention that, for $X$ as in Theorem 5.10, the Grothendieck group $\operatorname{KSp}(\mathcal{R}(X))$ and the Witt group $\mathrm{WSp}(\mathcal{R}(X))$ of symplectic spaces over $\mathcal{R}(X)$ are also computed in terms of $H_{\mathrm{alg}}^{k}$ in [10].

\section{References}

[1] R. Benedetti and M. Dedò, Counterexamples to representing homology classes by real algebraic subvarieties up to homeomorphism, Compositio Math. 53 (1984), 143-151.

[2] R. Benedetti and A. Tognoli, Approximation theorems in real algebraic geometry, Boll. Un. Mat. Ital. Suppl. 1980, no. 2, 209-228.

[3] R. Benedetti and A. Tognoli, On real algebraic vector bundles, Bull. Sci. Math. (2) 104 (1980), 89-112.

[4] R. Benedetti and A. Tognoli, Remarks and counterexamples in the theory of real algebraic vector bundles and cycles, in: Géométrie algébrique réelle et formes quadratiques, Lecture Notes in Math. 959, Springer, Berlin, 1982, 198-211.

[5] E. Bierstone and P. D. Milman, Canonical desingularization in characteristic zero by blowing up the maximum strata of a local invariant, Invent. Math. 128 (1997), 207-302.

[6] J. Bochnak, M. Coste and M.-F. Roy, Géométrie algébrique réelle, Ergeb. Math. Grenzgeb. (3) 12, Springer, Berlin, 1987. 
[7] J. Bochnak and J. Huisman, When is a complex elliptic curve the product of two real algebraic curves?, Math. Ann. 293 (1992), 469-474.

[8] J. Bochnak and W. Kucharz, Algebraic approximation of mappings into spheres, Michigan Math. J. 34 (1987), 119-125.

[9] J. Bochnak and W. Kucharz, Algebraic models of smooth manifolds, Invent. Math. 97 (1989), 585-611.

[10] J. Bochnak and W. Kucharz, K-theory of real algebraic surfaces and threefolds, Math. Proc. Cambridge Philos. Soc. 106 (1989), 471-480.

[11] J. Bochnak and W. Kucharz, Algebraic cycles and approximation theorems in real algebraic geometry, Trans. Amer. Math. Soc. 337 (1993), 463-472.

[12] J. Bochnak and W. Kucharz, Real algebraic hypersurfaces in complex projective varieties, Math. Ann. 301 (1995), 381-397.

[13] J. Bochnak, W. Kucharz and M. Shiota, The divisor class groups of some rings of global real analytic, Nash or regular functions, in: Géométrie algébrique réelle et formes quadratiques, Lecture Notes in Math. 959, Springer, Berlin, 1982, 218-248.

[14] J. Bochnak, W. Kucharz and M. Shiota, On algebraicity of global real analytic sets and functions, Invent. Math. 70 (1982), 115-156.

[15] A. Borel and A. Haefliger, La classe d'homologie fondamentale d'un espace analytique, Bull. Soc. Math. France 89 (1961), 461-513.

[16] N. Bourbaki, Algèbre commutative, Hermann, Paris, 1961-1965.

[17] L. Bröcker, Reelle Divisoren, Arch. Math. (Basel) 35 (1980), 140-143.

[18] W. Fulton, Intersection Theory, Ergeb. Math. Grenzgeb. (3) 2, Springer, Berlin, 1984.

[19] J. van Hamel, Real algebraic cycles on complex projective varieties, Math. Z. 225 (1997), $177-198$.

[20] H. Hir onaka, Resolution of singularities of an algebraic variety over a field of characteristic zero, Ann. of Math. (2) 79 (1964), 109-203, 205-326.

[21] S. T. Hu, Homotopy Theory, Academic Press, New York, 1959.

[22] J. Huisman, Real abelian varieties with complex multiplication, Ph.D. Thesis, Vrije Universiteit Amsterdam, 1992.

[23] J. Huisman, The underlying real algebraic structure of complex elliptic curves, Math. Ann. 294 (1992), 19-35.

[24] J. Huisman, A real algebraic vector bundle is strongly algebraic whenever its total space is affine, in: Real Algebraic Geometry and Topology, Contemp. Math. 182, Amer. Math. Soc., Providence, 1995, 117-119.

[25] F. Ischebeck and H.-W. Schülting, Rational and homological equivalence for real cycles, Invent. Math. 94 (1988), 307-316.

[26] N. Ivanov, Approximation of smooth manifolds by real algebraic sets, Russian Math. Surveys 37 (1982), 1-59.

[27] W. Kucharz, On homology of real algebraic sets, Invent. Math. 82 (1985), 19-25.

[28] W. Kucharz, Algebraic equivalence and homology classes of real algebraic varieties, Math. Nachr. 180 (1996), 135-140.

[29] F. Mangolte, Cycles algébriques sur les surface K3 réelles, Math. Z. 225 (1997), 559-576.

[30] F. Mangolte and J. van Hamel, Algebraic cycles and topology of real Enriques surfaces, Compositio Math. 110 (1998), 215-237.

[31] J.-J. Risler, Sur l’homologie des surfaces algébriques réelles, in: Géométrie algébrique réelle et formes quadratiques, Lecture Notes in Math. 959, Springer, Berlin, 1982, 381-385.

[32] H.-W. Schülting, Algebraische und topologische reelle Zyklen unter birationalen Transformationen, Math. Ann. 272 (1985), 441-448. 
[33] M. Shiota, Real algebraic realization of characteristic classes, Publ. Res. Inst. Math. Sci. 18 (1982), 995-1008.

[34] M. Shiota, Equivalence of differentiable functions, rational functions and polynomials, Ann. Inst. Fourier (Grenoble) 32 no. 4 (1982), 167-204.

[35] R. Silhol, $A$ bound on the order of $H_{n-1}^{(a)}(X, \mathbb{Z} / 2)$ on a real algebraic variety, in: Géométrie algébrique réelle et formes quadratiques, Lecture Notes in Math. 959, Springer, Berlin, 1982, 443-450.

[36] R. Silhol, Real Algebraic Surfaces, Lecture Notes in Math. 1392, Springer, Berlin, 1989.

[37] P. Teichner, 6-dimensional manifolds without totally algebraic homology, Proc. Amer. Math. Soc. 123 (1995), 2909-2914.

[38] R. Thom, Quelques propriétés globales des variétés différentiables, Comment. Math. Helv. 28 (1954), 17-86.

[39] A. Tognoli, Su una congettura di Nash, Ann. Scuola Norm. Sup. Pisa Cl. Sci. (3) 27 (1973), $167-185$.

[40] E. Witt, Zerlegung reeler algebraischer Funktionen in Quadrate, Schiefkörper über reellen Funktionenkörper, J. Reine Angew. Math. 171 (1934), 4-11. 